EPJ Web of Conferences 73, 07006 (2014)

DOI: $10.1051 /$ epjconf/20147307006

(C) Owned by the authors, published by EDP Sciences, 2014

\title{
The PRad experiment and the proton radius puzzle
}

\author{
Ashot Gasparian ${ }^{1,2, a}$ \\ ${ }^{1}$ NC A\&T State University, Greensboro NC, USA \\ ${ }^{2}$ For the PRad Collaboration at JLab
}

\begin{abstract}
New results from the recent muonic hydrogen experiments seriously questioned our knowledge of the proton charge radius, $r_{p}$. The new value, with its unprecedented less than sub-percent precision, is currently up to eight standard deviation smaller than the average value from all previous experiments, triggering the well-known "proton charge radius puzzle" in nuclear and atomic physics. The PRad collaboration is currently preparing a novel, magnetic-spectrometer-free ep scattering experiment in Hall B at JLab for a new independent $r_{p}$ measurement to address this growing "puzzle" in physics.
\end{abstract}

\section{Introduction}

The proton charge radius $\left(r_{p}\right)$ is one of the fundamental quantities in physics. Precise knowledge of its value is critically important for the understanding of the underlying quark-gluon structure of the nucleon in the theory of strong interactions - QCD. The precise determination of $r_{p}$ is equally critical for atomic physics - especially in the spectroscopy of atomic hydrogen. In the past more than four decades three major methods have been developed and used to measure $r_{p}$ : (i) The ep elastic scattering method, in which the slope of the extracted electric form factor, $G_{E}^{p}$, at low $Q^{2}$ defines the rms radius of the proton, $r_{p}$. The average value of $r_{p}$ for this method, given by a model independent analysis of electron scattering data is $r_{p}=0.870(26) \mathrm{fm}$ [1]. Several known experimental factors limit the precision in these experiments, and the typical uncertainties of individual experiments currently are at the level of $2 \%$. Recently, a new state-of-the-art experiment was performed at MAMI Mainz giving $r_{p}=0.879(8) \mathrm{fm}$ [2], and it is consistent with previous ep scattering results. (ii) The spectroscopy of electronic (ordinary) hydrogen atom through the Lamb shift measurements defines the proton radius, $r_{p}$. The value of $r_{p}$ from this method is currently consistent with the ep scattering results: $r_{p}=0.8775(51) \mathrm{fm}$ [3]. (iii) Recently developed method using the Lamb shift measurements from the muonic hydrogen. The muonic hydrogen result $\left(r_{p}=0.8409(4) \mathrm{fm}\right)[4,5]$, with its unprecedented less than $0.1 \%$ precision, is currently up to eight standard deviation smaller than the average value from all previous experiments, triggering the well-known "proton radius puzzle" in nuclear and atomic physics. So far, all theoretical efforts and more precise simulations failed to explain this discrepancy on the value of a fundamental quantity $-r_{p}$. The current situation critically requires performing new

\footnotetext{
ae-mail: agaspari@ncat . edu
}

This is an Open Access article distributed under the terms of the Creative Commons Attribution License 4.0, which permits unrestricted use, distribution, and reproduction in any medium, provided the original work is properly cited. 
high precision and high accuracy experiments. The PRad collaboration is currently preparing a new magnetic-spectrometer-free ep scattering experiment in Hall B at Jefferson Lab for a new independent measurement of $r_{p}$ to address this recently growing "puzzle" in hadronic physics.

\section{The PRad experiment at JLab}

Practically all previous ep scattering experiments have been done using the traditional magnetic spectrometer method. The PRad experiment is designed to use a calorimetric method for the first time in these measurements. We will use the PrimEx HyCal high resolution and large acceptance electromagnetic calorimeter to detect the forward scattered electrons from the target hydrogen, as well as, both electrons from the Møller scattering process. Also, a windowless hydrogen gas flow target will be implemented in this experiment which will be a first in these type of experiments [6]. With that, the PRad experiment will have three major improvements over previous ep scattering experiments: (i) It will have a good possibility to reach the extreme forward angles $\left(\sim 0.7^{\circ}-6^{\circ}\right)$ for the first time in ep scattering experiments. This will allow to reach extremely low $Q^{2}$ range $\left(10^{-4}-10^{-2}\right)(\mathrm{GeV} / \mathrm{c})^{2}$ for few $\mathrm{GeV}$ incident electron beams. The lowest $Q^{2}$ range measured up to date is in the recent Mainz experiment [2] where the minimum value for $Q^{2}$ reached was $3 \times 10^{-3}(\mathrm{GeV} / \mathrm{c})^{2}$. Reaching low $Q^{2}$ range is critically important since the rms charge radius of the proton is extracted as the slope of the measured electric form factor $-G_{E}^{p}\left(Q^{2}\right)$ at the $Q^{2}=0$ point. By continuously measuring the Møller scattering process, we will control the uncertainties in the extracted cross sections at very low $Q^{2}$ range, in particular. We will also take data for two different energy values for the incident electron beam to cover a sufficient range in $Q^{2}\left(10^{-4}-10^{-2}\right)(\mathrm{GeV} / \mathrm{c})^{2}$ to facilitate the extraction of the slope from $G_{E}^{p}$ vs. $Q^{2}$ dependence. (ii) The ep cross sections in this experiment will be normalized to the well known QED process - Møller scattering, which will be measured simultaneously during the experiment within the same detector acceptance. This, arguably, will be a superior method to control the systematic uncertainties in the $e p \rightarrow e p$ cross sections. (iii) The novel windowless hydrogen target will significantly reduce the experimental background from the target windows, which was one of the typical sources of systematic uncertainties for all previous experiments.

Simultaneous detection of both $e p \rightarrow e p$ and $e^{-} e^{-} \rightarrow e^{-} e^{-}$processes will directly relate the $e p$ cross sections to the $e^{-} e^{-}$Møller cross sections, which can be calculated with sub-percent accuracy $(\sim 0.5) \%$ within the QED framework, including the radiative corrections:

$$
\left(\frac{d \sigma}{d \Omega}\right)_{e p}\left(Q_{i}^{2}\right)=\left[\frac{N_{\exp }^{\mathrm{yield}}\left(e p \rightarrow e p \operatorname{in} \theta_{i} \pm \Delta \theta\right)}{N_{\exp }^{\mathrm{yield}}\left(e^{-} e^{-} \rightarrow e^{-} e^{-}\right)} \cdot \frac{\varepsilon_{\mathrm{geom}}^{e^{-} e^{-}}}{\varepsilon_{\mathrm{geom}}^{e p}} \cdot \frac{\varepsilon_{\mathrm{det}}^{e^{-}}}{\varepsilon_{\mathrm{det}}^{e p}}\right]\left(\frac{d \sigma}{d \Omega}\right)_{e^{-} e^{-}} .
$$

With this method, the two major sources of systematic uncertainties, the number of beam electrons and the number of protons in the target, which dominated in all previous scattering experiments, are not contributing factors in our experiment. The contributions of two other sources of systematic uncertainties, the geometrical acceptances $\left(\varepsilon_{\mathrm{geom}}^{e^{-} e^{-}} / \varepsilon_{\mathrm{geom}}^{e p}\right)$ and the detection efficiencies $\left(\varepsilon_{\mathrm{det}}^{e^{-} e^{-}} / \varepsilon_{\operatorname{det}}^{e p}\right)$ will also cancel out in their ratios to first order.

\section{PRad experimental setup}

The PRad experimental apparatus consist of the following main elements [6] (see Fig. 1): (i) windowless hydrogen gas flow target; (ii) a vacuum box with a single thin window at the calorimeter only, spanning $\sim 5 \mathrm{~m}$, with a target side opening of $0.3 \mathrm{~m}$ diameter and a $1.7 \mathrm{~m}$ diameter thin $\mathrm{Al}$ window at the front of HyCal. The Al window will have a thickness of $2.5 \mathrm{~mm}$, with a $4 \mathrm{~cm}$ diameter central portion thinned to about $200 \mu \mathrm{m}$ or, if possible, a thin Kapton window for the passage of electron beam; (iii) scintillating $X-Y$ veto counters to eliminate the neutral background; and (iv) high resolution, large acceptance PrimEx HyCal electromagnetic calorimeter. It is planned to run this experiment in 


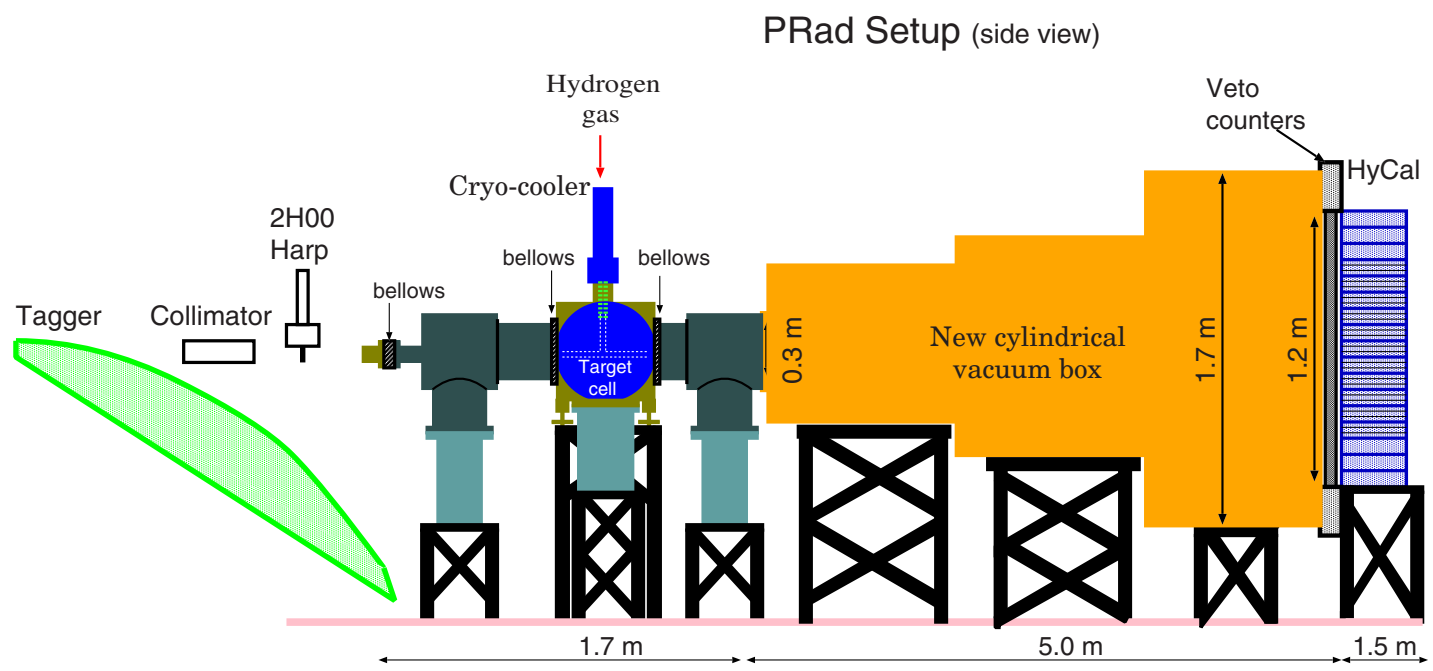

Figure 1. Schematic layout of the PRad experimental setup (not to scale).

Hall B at JLab, therefore, the experiment will also use the standard Hall B beam line equipment, designed for low intensity electron beam current (0.1-10 nA, and the standard Hall B photon Tagger facility for the calibration of the HyCal calorimeter and related detectors before and after the physics data taking periods. A novel addition in this experiment, other than the calorimetry method, is the usage of a windowless hydrogen gas flow target having a thickness of about $\sim 1 \times 10^{18}$ hydrogen atoms $/ \mathrm{cm}^{2}$. With the planned incident electron beam current of $10 \mathrm{nA}$, this gives a luminosity of about $\mathcal{L} \approx 6 \times 10^{28} \mathrm{~cm}^{-2} \mathrm{~s}^{-1}$. This is a relatively high density for the typical windowless gas flow targets, used before mostly in collider experiments. This high density will be reached by flowing cryocooled hydrogen gas $\left(\right.$ at $\left.25^{\circ} \mathrm{K}\right)$ through a small diameter $(8 \mathrm{~mm})$ thin-walled cylindrical pipe $(4 \mathrm{~cm}$ in length) open at both ends. This novel target is expected to significantly reduce the experimental background in our experiment. The incident electrons with $1.1 \mathrm{GeV}$ (and $2.2 \mathrm{GeV}$ ) energies will scatter off a windowless cryo-cooled hydrogen gas flow target and will be detected with the high resolution PrimEx HyCal calorimeter. The Møller - scattered electrons from the atomic electrons in hydrogen, together with the recoiling electrons will also be detected in the calorimeter within the same geometrical acceptance and similar kinematics. An $X-Y$ scintillator veto detector will be installed in front of HyCal to suppress neutral particle backgrounds in the experiment. A new vacuum box will be installed between the target chamber system and the veto counters. The PrimEx HyCal calorimeter [7] is a hybrid electromagnetic calorimeter consisting of two different type of shower detectors, $576 \mathrm{~Pb}$-glass modules $\left(4.0 \times 4.0 \times 45 \mathrm{~cm}^{3}\right)$ and $1152 \mathrm{PbWO}_{4}$ crystal modules $\left(2.05 \times 2.05 \times 18.0 \mathrm{~cm}^{3}\right)$. The calorimeter with its cross sectional area of $118 \times 118 \mathrm{~cm}^{2}$ will be located in the beam line at a distance of $\sim 5 \mathrm{~m}$ from the target, which will provide a geometrical acceptance of $\sim 25 \mathrm{msr}$ in this experiment. The electron beam will pass through a $4.0 \times 4.0 \mathrm{~cm}^{2}$ hole in the central part of $\mathrm{HyCal}\left(2 \times 2 \mathrm{PbWO}_{4}\right.$ modules are removed from the assembly) allowing a direct and simultaneous detection of both ep $\rightarrow$ ep and $e^{-} e^{-} \rightarrow e^{-} e^{-}$processes in this experiment. The collaboration is currently exploring possibilities to add $X$-, $Y$-coordinate detectors just in front of the HyCal calorimeter to: (i) improve the coordinate reconstruction by a factor of ten for the crystal part (similar improvement in $Q^{2}$ resolution); (ii) make an unbiased coordinate reconstruction, including the transition region between the $\mathrm{PbWO}_{4}$ and $\mathrm{Pb}-$ glass detectors; (iii) improve the coordinate resolution by a factor of twenty for the $\mathrm{Pb}$-glass part which dramatically increases the $Q^{2}$ coverage in the experiment in one experimental setting. 


\section{Expected results and uncertainties}

The ep elastic cross sections will be measured by normalizing them to the Møller cross sections, which will be obtained from theoretical QED calculations within the same acceptances taking into account the radiative effects. At this low $Q^{2}$ range, the contribution from the magnetic form factor can be neglected within the accuracy of this experiment. Thus, the electric form factor, $G_{E}^{p}$, can be extracted from the measured $e p$ elastic cross sections $v s . Q^{2}$. The slope of $G_{E}^{p} v s . Q^{2}$ at this very low $Q^{2}$ range will determine the rms proton charge radius, $r_{p}$. Full Monte Carlo simulations have been performed to estimate the uncertainties in the extraction of $r_{p}$ taking into account the realistic geometry of the experiment, detector resolutions and radiative effects. Both ep and $e^{-} e^{-}$Møller processes are the two electromagnetic processes at these forward angles with the highest rates in this energy range. We expect to have enough statistics within the approved beam time (15 days) to provide statistical uncertainties at the level of $0.2 \%$ in the extracted $r_{p}$. The control of the systematic uncertainties has always been one of the most challenging problems in past $e p$-scattering experiments. Our novel experiment with its: (i) possibility to reach very low $Q^{2}$ range; (ii) normalizing the ep cross sections to the Møller scattering; and (iii) windowless hydrogen gas target to minimize the physics background processes, is, arguably, the most optimized ep measurement to reach a sub-percent systematic uncertainties in the extraction of $r_{p}$. Analyzing the remaining sources of the systematic uncertainties in our experiment, we estimate $0.4 \%$ total systematic uncertainty. Combining that with the $0.2 \%$ statistical uncertainties, we project to have the total uncertainty on the $r_{p}$ extraction at the level of $0.6 \%$.

\section{Summary}

The PRad collaboration is preparing a novel magnetic-spectrometer-free ep scattering experiment to extract the proton charge radius in Hall B at Jefferson Lab. This experiment will critically improve all systematic uncertainties typical for the traditional magnetic spectrometer experiments by: (i) normalizing the ep cross sections to the well known QED process - Møller scattering; (ii) achieving very forward scattering angles for the first time in ep experiments while keeping the $Q^{2}$ range $\left(10^{-4}-10^{-2}\right.$ $\left.(\mathrm{GeV} / \mathrm{c})^{2}\right)$ large enough for the practically model independent extraction of $r_{p}$; (iii) using a windowless hydrogen gas flow target to sufficiently reduce the experimental background typical for all previous $e p$ experiments. PRad, with its sub-percent precision, is expected to have a direct impact on the "proton radius puzzle" currently developing in hadronic physics.

This project is supported in part by the USA NSF MRI award PHY-1229153 and NSF award PHY-1205962.

\section{References}

[1] R.J. Hill, G. Paz, Phys. Rev. D 82, 113005 (2010)

[2] J.C. Bernauer et al., Phys. Rev. Lett. 105, 242001 (2010)

[3] P.J. Mohr, B.N. Taylor, D.B. Newell, Rev. Mod. Phys. 84, 1527 (2012)

[4] R. Pohl et al., Nature 466, 213 (2010)

[5] A. Antognini et al., Science 339, 417 (2013)

[6] A. Gasparian, M. Khandaker, H. Gao, D. Dipangkar et al., JLab Proposal E12-11-106. (http://www.jlab.org/exp_prog/proposals/11/PR12-11-106.pdf)

[7] M. Kubantsev et al., AIP Conf. Proc. 867, 51 (2006) 